Revue internationale de l'économie sociale

Recma

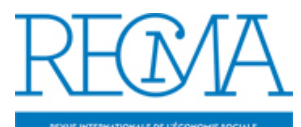

\title{
En bref
}

\section{Jordane Legleye}

Numéro 331, janvier 2014

URI : https://id.erudit.org/iderudit/1023481ar

DOI : https://doi.org/10.7202/1023481ar

Aller au sommaire du numéro

Éditeur(s)

Association Recma

ISSN

1626-1682 (imprimé)

2261-2599 (numérique)

Découvrir la revue

Citer ce document

Legleye, J. (2014). En bref. Revue internationale de l'économie sociale, (331),

20-26. https://doi.org/10.7202/1023481ar

Ce document est protégé par la loi sur le droit d'auteur. L’utilisation des services d'Érudit (y compris la reproduction) est assujettie à sa politique d'utilisation que vous pouvez consulter en ligne.

https://apropos.erudit.org/fr/usagers/politique-dutilisation/
Cet article est diffusé et préservé par Érudit.

Érudit est un consortium interuniversitaire sans but lucratif composé de l’Université de Montréal, l'Université Laval et l'Université du Québec à Montréal. Il a pour mission la promotion et la valorisation de la recherche. https://www.erudit.org/fr/ 


\section{EN BREF}

\section{L’économie sociale}

Les ambitions internationales de l'ESS Du 9 au 11 novembre 2013, le Forum international de dirigeants de l'économie sociale et solidaire (Rencontres du MontBlanc [RMB]) a réuni 350 participants, provenant de quarante-cinq pays des cinq continents, sur le thème " Changer le cap de la mondialisation avec l'économie sociale et solidaire: vers des objectifs du millénaire pour le développement (OMD) post-2015 ». Ces rencontres ont été placées sous le haut patronage du président de la République française, qui a décidé d'engager le pays dans le soutien à la création du comité de pilotage international de l'ESS, appelé leading group au sein des Nations unies. Composé d'Etats, d'institutions internationales et d'acteurs de l'ES, "[celui-ci] sera un interlocuteur privilégié pour assurer la prise en compte d'une forme d'économie participative, juste et durable, à l'occasion des grands sommets internationaux ». La déclaration finale des RMB évoque notamment la conception d'une cartographie mondiale des initiatives d'ESS; le développement "des projets transfrontières de réappropriation des biens communs, [par exemple] la réappropriation des terres par les agriculteurs"; l'engagement d'une concertation pour "créer un fonds de garantie internationale de l'ESS »; la planification d'actions communes avec la Confédération internationale des syndicats et la Confédération européenne des syndicats; la création "d'une école mondiale de l'ESS sur le net en partenariat avec l'académie de l'ESS de Turin, avecl'Unrisd, l'Institut panafricain de développement (IPD), etc. ». Rappelons que l'ONU a créé en son sein, le 30 septembre 2013, une agence interinstitutionnelle réunissant les FAO, l'Unesco, l'Unaids, l'UN Women... Il s'agit de la première reconnaissance institutionnelle du secteur au niveau international (www. recma.org/node/3746).

En savoir plus: http://recma.org/node/3747.

\section{Coopmed : 10 millions d'euros pour financer l'ESS \\ de la Méditerranée sud}

Les acteurs privés n'ont pas attendu ce leading group pour lancer en janvier Coopmed, "afin de participer au financement et à l'accompagnement des acteurs de l'économie sociale». Le Crédit coopératif, la Macif, la Banque européenne d'investissement (BEI) et des financeurs des deux rives de la Méditerranée ont ainsi dédié 10 millions d'euros pour des projets d'ESS au Maroc, en Tunisie, au Liban et en Turquie. En savoir plus: www.recma.org/node/3799.

\section{Naissance du Collectif algérien pour le développement de l'ESS}

En écho, le Collectif algérien pour le développement de l'économie sociale et solidaire (Cadess) a été créé, le 27 novembre, au terme d'une journée organisée par un collectif d'associations algériennes à l'assemblée populaire de la wilaya de Tizi-Ouzou, avec le soutien de la Macif.

En savoir plus: www.recma.org/node/3775 et « Le rôle de l'Etat dans l'économie sociale en Algérie », par C. Mustapha et B. Abdellah, Recma, $\mathrm{n}^{\circ} 321$, www.recma.org/node/1360.

\section{Le marché de l'emploi cadre dans l'ESS}

L’Apec a publié fin 2013 une étude réalisée à partir de 12600 offres d'emploi de cadre diffusées en 2012 par 2500 entreprises de l'ESS. Le secteur représente près de $13 \%$ de l'emploi cadre du secteur privé en France. Avec un taux d'encadrement moyen de $15 \%$ (21\% dans les mutuelles, $14 \%$ dans les associations), la part des cadres dans l'ESS est proche de celle des cadres dans l'ensemble du secteur privé. Les associations ont diffusé 
$64 \%$ des offres, les coopératives $18 \%$, les mutuelles $13 \%$ et les fondations $5 \%$. Les offres d'emploi en gestion-financeadministration représentent $21 \%$ des offres de l'ESS, soit deux fois plus que l'ensemble des offres Apec. Les coopératives se singularisent avec une part d'offres parues supérieure dans la fonction financetrésorerie $(8 \%$, contre seulement $1 \%$ pour l'ensemble des offres). Pour les mutuelles, la majorité des postes de cadres à pourvoir se trouvent en droit-fiscalité ou en administration-gestion organisation. Si les associations et les fondations se distinguent avec une part élevée d'offres publiées en ressources humaines (respectivement 14 et $10 \%$, contre $3 \%$ pour l'ensemble des offres), les mutuelles et surtout les coopératives ont en revanche principalement des postes de commerciaux et de marketing à pourvoir (respectivement 30 et $48 \%$, contre seulement $23 \%$ pour l'ensemble des offres). Le salaire médian brut annuel proposé dans les offres d'emploi de l'ESS publiées en 2012 est de 35000 euros ( 37500 en moyenne dans le reste du secteur privé). Pour les associations, qui représentent la majorité des offres publiées dans l'ESS, le niveau moyen de rémunération proposé est plus faible (34500 euros). Si la majorité des entreprises de l'ESS sont des associations de petite taille, ce secteur se distingue néanmoins avec une surreprésentation des offres d'emploi de cadre diffusées par des grandes entreprises: une offre sur deux provient d'une entreprise de plus de 250 salariés, contre seulement une sur trois pour l'ensemble du privé. Les coopératives offrent plus de postes pour les jeunes diplômés (une offre sur deux) que le reste du secteur privé, et $40 \%$ des offres sont concentrées en Ile-de-France.

En savoir plus: www.recma.org/node/3762.

\section{Rapport Combrexelle:}

la représentativité patronale en débat Alors que Michel Sapin, ministre du Travail, envisage de ne pas organiser les élections prud'homales de 2015 (100 millions d'euros d'économie sur le dos de la démocratie sociale...), son ministère a rendu public fin 2013 le rapport de Jean-Denis Combrexelle, directeur général du travail, sur la « réforme de la représentativité patronale »: primat de la branche sur l'interprofessionnalité; réduction drastique du nombre de branches (près de 1000 aujourd'hui à 100 dans dix ans); alignement des modalités de validation d'accords sur les règles des syndicats de salariés (signature possible d'un accord si le syndicat représente $30 \%$ de « l'audience pondérée » et en cas d'absence "droit d'opposition majoritaire " d'un syndicat représentant $50 \%$...). Principal motif de satisfaction pour les syndicats d'employeurs du secteur, le rapport préconise de les intégrer au Conseil supérieur de la prud'homie, à la Commission nationale de la négociation collective et au Conseil national de l'emploi. En savoir plus: www.recma.org/node/3789.

\section{Loi-cadre ESS, et après?}

En attendant d'être votée à l'Assemblée nationale, la discussion sur la loi-cadre ESS est menée hors du Parlement. Le catalogue très technique des mesures adoptées par le Sénat début novembre 2013 a désormais laissé place à des discussions sur le fond, en un mot "idéologiques". La tribune d'Emmanuel Antoine (Minga) et Stéphane Veyer (Coopaname), initialement intitulée "Adieu à l'ES » et publiée dans Le Monde du 21 novembre sous le titre « Pour une ESS de combat", a ouvert le bal. "Voilà, c'est fait. L'économie sociale et solidaire voulait être "reconnue", elle l'est. Elle souhaitait changer d'échelle: elle en aura les moyens. Les entrepreneurs sociaux voulaient qu'on leur fasse une place sur la photo de famille: ils sont au premier rang. [...] Doit-on par exemple se réjouir de la création de grands groupes associatifs recourant aux techniques, aux personnels, aux logiques de l'entreprise cotée pour asseoir leurs capacités? Doit-on se féliciter du développement du microcrédit ou des fondations d'entreprise? Doit-on se satisfaire de l'application de principes 
gestionnaires aux rapports entre pouvoirs publics et monde non lucratif? [...] L'enjeu n'est donc ni un problème de taille, ni de reconnaissance institutionnelle, mais la cruelle absence d'un projet politique commun à ces organisations qui pourrait irriguer l'économie toute entière. [...] A l'ESS "des bonnes causes", nous appelons donc à substituer une ESS "de combat". Voilà notre programme. Il a le mérite de la simplicité. Il consiste à amener partout, dans tous les recoins de l'économie, à toutes les échelles, un questionnement légitime sur le pouvoir, la propriété, le savoir. [...] Il est temps de dire adieu à l'ESS en tant que rassemblement hétéroclite "d'entreprises sympas", pour faire vivre un projet politique porté bien au-delà de nos organisations par toutes celles et ceux qui veulent changer de société; cesser de nous tourner constamment vers la puissance publique et enfin s'adresser au monde du travail » (www.recma.org/ node/3757).

Dans L'Humanité du 7 janvier, Anne-Laure Desgris et Stéphane Veyer posent "avec malice" la question essentielle du sens politique de l'ESS: «Si [cette dernière] existe, n'est-cepasjustement pour refouler la question de l'idéologie, du projet politique qui devrait normalement se loger au cœur de toute initiative associative, coopérative ou mutualiste?» Et les auteurs de conclure sur la possibilité d'une autre politique publique en faveur de l'ESS: " Favoriser l'ESS ne devrait pas consister à développer les entreprises existantes, mais à donner du pouvoir aux groupements de personnes. Radicalement. Obstinément. Systématiquement. Autrement dit: poser en permanence la question de la citoyenneté, de la démocratie, de l'autogestion dans toutes les politiques publiques. On redécouvrirait alors que la première vocation de l'ESS n'est pas de suppléer l'Etat, mais de produire par elle-même du droit, des droits, et d'œuvrer à l'émancipation des individus. "

Tribune isolée de francs-tireurs partisans? Le 9 novembre, l'économiste Hervé Defalvard estimait de manière plus académique que, "pour définir l'ESS, la loi a davantage écouté la pensée des écoles de commerce que les économistes" (www.recma.org/node/3765). Sa collègue Nadine Richez-Battesti rappelle justement, dans L'Humanité du 7 janvier, que "les différentes organisations qui composent l'ESS ont émergé du mouvement social il y a près de deux siècles, en réaction aux effets pervers du système capitaliste. Ces organisations ne se limitent pas à leur finalité sociale et à la gestion des plus démunis. Elles proposent une articulation renouvelée entre l'économique (la production de biens et services dans le cadre d'une propriété collective), le social (finalité sociale et solidarité) et le politique (la démocratie économique dans les organisations). C'est la combinaison des trois dimensions qui fonde l'ES » et résume le "principe de double qualité des membres, qui sont à la fois bénéficiaires de l'action et propriétaires collectifs». Une loi-cadre n'épuisera donc pas le débat. D'autant que Sofia Arana Landin rappelait au colloque du Ciriec à Anvers, du 24 au 26 octobre, qu'aucun décret d'application de la loi-cadre espagnole adoptée en 2011 (Recma, $\mathrm{n}^{\circ} 321$ ) n'avait encore été publié...

La campagne d'interpellation des élus locaux lancée par l'association Agir pour une économie équitable (Ap2E) sur quatre articles de la loi française se révèle donc très opportune dans le contexte électoral chargé de 2014 (art. 11 et 12 sur la transmission d'entreprise à leurs salariés; art. 14 sur la révision coopérative; art. 35 sur la gouvernance des mutuelles; www.recma.org/node/3816).

En savoir plus: www.recma.org/node/3841.

\section{La coopération}

\section{L’ACI invite les coopérateurs à définir les principes coopératifs}

Du $1^{\text {er }}$ au 5 novembre 2013, le Cap (Afrique du Sud) a accueilli la conférence mondiale et l'assemblée générale de l'Alliance coopérative internationale (ACI). L'occasion pour cette dernière de remettre le Rochdale Pioneers Award à la fondation anglaise Plunkett. Elle y a également dévoilé sa nouvelle identité visuelle. Pauline Green a été réélue présidente 
et dix-neuf nouvelles personnalités ont effectué leur entrée au conseil d'administration de l'ACI, dont Jean-Louis Bancel, président du Groupe Crédit coopératif. Celui-ci est également président du comité des principes de l'ACI, qui invite, conformément au Plan d'action pour une décennie coopérative ("Actualité», Recma, $n^{\circ} 326$ ), les coopérateurs du monde entier à apporter leur contribution en vue d'élaborer une ébauche de notes d'orientation pour les principes coopératifs. Ces principes ont été établis en 1937, puis révisés en 1966 et en 1995 pour concilier la doctrine coopérative et les véritables conditions d'existence des coopératives. Lébauche des notes d'orientation pour les principes III (participation économique des membres), $\mathrm{V}$ (éducation, formation et information) et VII (engagement envers la communauté) est disponible et amendable en ligne jusqu'au 15 mai 2014.

En savoir plus: www.recma.org/node/3824.

\section{La FAO élargit son soutien aux coopératives agricoles}

Le protocole d'accord signé le 15 octobre dernier entre la FAO (Organisation des Nations unies pour l'alimentation et l'agriculture) et l'Alliance coopérative internationale qualifie d'« unique » la contribution des coopératives agricoles reconnues comme "des partenaires cruciaux dans la lutte contre la faim et la pauvreté». Le protocole renforce le rôle des coopératives agricoles dans les négociations avec les partenaires publics, les ONG et le secteur agroalimentaire. En mettant l'accent sur le partage des connaissances, il leur facilite l'accès aux capitaux et encourage le développement durable des entreprises au niveau tant environnemental et social qu'économique.

En savoir plus: www.recma.org/node/3713.

\section{Cuba: les coopératives agricoles d'Etat non rentables peu à peu dissoutes}

L'agriculture cubaine est à son tour atteinte par l'exigence de rentabilité. Depuis cinq ans, plus de 400 coopératives agricoles d'Etat ont été dissoutes, parce qu'elles ne parvenaient pas à s'autofinancer. Amorcé en 2008, le mouvement s'est accéléré depuis 2012. Ces unités de "base de production coopérative » n'exploitent plus que $28 \%$ des surfaces cultivables de l'île. Ces dernières années, les terres ont été redistribuées à 180000 agriculteurs, regroupés en coopératives indépendantes. Ils peuvent désormais vendre directement leur production, sans passer par les circuits de distribution étatiques. L'objectif du gouvernement cubain est de redynamiser le secteur agricole afin de réduire considérablement les importations ( $80 \%$ des produits consommés). En savoir plus: www.recma.org/node/3699 et Marzin J., 2013, « Les leçons et les enjeux du mouvement coopératif agraire à Cuba », Recma, $\mathrm{n}^{\circ} 328$.

\section{La France, deuxième puissance coopérative mondiale}

Selon le World Co-operative Monitor, observatoire mondial des coopératives, les trois cents premières coopératives et mutuelles affichent un chiffre d'affaires (CA) égal à l'économie de la neuvième économie mondiale. Ce classement concerne vingttrois pays et compte près de 2100 milliards de dollars de CA, en croissance depuis 2010 (1975 milliards de dollars US). Deux structures françaises figurent dans les dix plus grandes entreprises coopératives et mutualistes du monde (ACDlec-E. Leclerc, en septième position, avec un CA de 49,05 milliards de dollars, et le Groupe Crédit agricole, en neuvième position avec un chiffre d'affaires de 45,49 milliards de dollars). La France se place en deuxième position mondiale, ses coopératives réalisant 363,63 milliards de dollars de chiffre d'affaires, soit plus que la moitié du CA coopératif des Etats-Unis (662,23 milliards de dollars, mais pour une population cinq fois plus importante). Suivent, notamment, le Japon, l'Allemagne, les Pays-Bas, l'Italie, l'Espagne, la Suisse, le Royaume-Uni, la Finlande et le Canada.

En savoir plus: www.recma.org/node/3745. 


\section{La coopération agricole française monte en puissance}

Maïsadour, Terrena, Sodiaal, Vivescia... Ces noms sont peu familiers, et pourtant leurs marques se retrouvent au quotidien dans les assiettes des Français: Florette, Daddy, Yoplait, Delpeyrat, Francine, Labeyrie, Entremont... Les coopératives agricoles réalisent 84,3 milliards d'euros de chiffre d'affaires - soit $40 \%$ de celui de l'agroalimentaire français et plus 600 millions en un an - et emploient plus de 160000 personnes. Cependant, la coopération agricole française serait encore trop morcelée pour le marché mondial (2800 entreprises coopératives, unions et Sica en 2013, contre 2850 en 2012), a affirmé Coop de France lors de son congrès des 18 et 19 décembre. Pour Philippe Mangin, président de Coop de France, l'émergence d'un nouveau leader coopératif(Agrial) et le renforcement du premier groupe laitier (Sodiaal, qui a fusionné tout début janvier avec deux coopératives pour représenter $31 \%$ du marché des fromages en France) constituent "de bonnes nouvelles qui permettront d'équilibrer les négociations avec la grande distribution et de conquérir des places à l'export». En savoir plus:www.recma.org/node/3807.

\section{Les mutuelles}

\section{Guide de gouvernance}

\section{des coopératives et des mutuelles}

Un groupe de dirigeants de coopérative et de mutuelle s'est réuni sous la co-présidence d'Etienne Pflimlin, président d'honneur de la Confédération nationale du Crédit mutuel (CNCM), et de Daniel Lebègue, président de l'Institut français des administrateurs (IFA), pour mettre en commun les différentes pratiques de gouvernance et les différents modes de démocratie en œuvre dans leurs entreprises: processus démocratique, représentativité des administrateurs, bénévolat, fonctionnement des instances, prise de décision... En l'absence de document de référence commun, il est apparu important de mieux formaliser ces principes dans un guide de la gouvernance des coopératives et des mutuelles. Celui-ci rappelle la primauté du sociétariat et le rôle de l'administrateur-élu représentant les sociétaires. Il formule plusieurs recommandations pour organiser et responsabiliser le conseil et renforcer l'efficacité de ses travaux. Les dispositions du guide pourront faire l'objet d'adaptations et d'ajouts permettant de prendre en compte la diversité des situations rencontrées. Les sociétés coopératives et mutualistes qui se réfèreront à ce guide de gouvernement d'entreprise pourront en faire état dans leur document de référence ou dans leur rapport annuel.

En savoir plus: www.recma.org/node/3815.

\section{Les Scic, quelle opportunité}

\section{pour les groupements mutualistes?}

L'Agence de valorisation des initiatives socio-économiques (Avise), en partenariat avec l'Inter-réseaux Scic (société coopérative d'intérêt collectif), la Confédération générale des Scop et la Fédération nationale de la Mutualité française (FNMF), a publié fin 2013 un petit guide de présentation d'une vingtaine de Scic œuvrant aujourd'hui dans le domaine de la santé et de la prévention.

En savoir plus: www.recma.org/node/3817.

\section{Algérie : un projet de loi relative aux mutuelles sociales}

Le Conseil des ministres du 30 décembre 2013 a étudié un projet de loi relative aux mutuelles sociales, qui s'inscrit dans le cadre de la réforme globale du système national de Sécurité sociale. Il institue, au titre des prestations facultatives des mutuelles, des retraites complémentaires financées par les cotisations des adhérents. L'introduction de ce nouveau dispositif induit la précision du statut des mutuelles, devenant des personnes morales, ainsi que l'élargissement de leur champ d'intervention, à travers des prestations individuelles du régime général, supplémentaires à celles qui sont servies par la Sécurité sociale. Le 22 janvier, le comité 
de coordination des mutuelles appelait à surseoir à ce projet de loi élaboré « de façon unilatérale par l'administration ».

En savoir plus: www.recma.org/node/3830.

\section{Association pour l'accès}

à la complémentaire santé

\section{des populations en précarité}

Le relèvement de $8,3 \%$, en juillet 2013 , des plafonds d'attribution de la CMUC (l'assurance complémentaire gratuite liée à la couverture maladie universelle) et de l'aide financière à la complémentaire santé (ACS) accordée par la Sécurité sociale permet à plusieurs centaines de milliers de personnes d'accéder à une mutuelle. ATD-Quart Monde et les groupes mutualistes Adréa, Apréva, Eovi, Harmonie Ociane ont ainsi créé l'Association pour l'accès à la complémentaire santé des populations en précarité (ACS-P), qui promeut une nouvelle " garantie santé ACS " permettant une prise en charge des frais d'hôpital, d'obsèques, de lunettes, de dents, des vignettes oranges, etc., pour les populations vulnérables.

En savoir plus: www.recma.org/node/3849.

\section{Les modalités de fusion des sociétés d'assurance mutuelle}

Le décret $n^{\circ} 2014-12$ du 8 janvier 2014 relatif aux modalités de fusion des sociétés d'assurance mutuelle et modifiant certaines dispositions du chapitre III du titre I $^{\text {er }} \mathrm{du}$ livre VI du Code monétaire et financier vise à rendre applicable la procédure de fusion des sociétés anonymes qui réalisent des opérations d'assurance aux sociétés d'assurance mutuelle. Il précise notamment "l'affirmation du principe de transmission universelle de patrimoine de l'absorbée vers l'absorbante" et l'obligation d'" approbation du projet de fusion par les assemblées générales des sociétés concernées ». Le Code des assurances s'enrichit d'une sous-section $\mathrm{n}^{\circ} 9$ "Fusion de sociétés d'assurance mutuelle ", contenant les nouveaux articles R. 322-106-2 à 322-106-11.

En savoir plus: www.recma.org/node/3847.
Mutuelle santé d'entreprise: fin de l'exonération de l'impôt sur le revenu

L'article 4 de la loi de finances pour 2014 publiée le 30 décembre dernier au Journal officiel prévoit la suppression de l'exonération fiscale des cotisations de couverture complémentaire santé prises en charge par l'employeur. Le gouvernement a en effet décidé de supprimer l'exonération fiscale du salarié sur la participation de l'employeur aux contrats collectifs de complémentaire santé, au motif que l'abondement de l'employeur serait assimilable à un avantage en nature, donc, à ce titre, à un complément de salaire taxable. Recette attendue pour l'Etat: 960 millions d'euros en 2014, selon les prévisions budgétaires de Bercy; une hausse d'impôt estimée entre 90 et 150 euros en moyenne par le Centre technique des institutions de prévoyance (CTIP) pour les titulaires de contrat santé collectif et obligatoire. Les trois autres risques pouvant être couverts par la prévoyance complémentaire (incapacité de travail, invalidité et décès) ne sont pas concernés.

En savoir plus www.recma.org/node/3831.

\section{Les associations}

\section{Allégement de la taxe sur les salaires}

Le crédit d'impôt compétitivité emploi (Cice; "En bref», Recma, $n^{\circ}$ 327) annoncé en novembre 2012 ne concerne pas, par définition, les associations non soumises à l'impôt sur les sociétés (seules $13 \%$ des associations y sont assujetties). Le plafond d'abattement de la taxe sur les salaires a donc été remonté de 6000 à 20000 euros pour les petites associations. A compter du $1^{\text {er }}$ janvier 2014, $70 \%$ des associations employeuses économiseront 14000 euros. Reste que cet abattement est forfaitaire, alors que le Cice croît en fonction de l'augmentation de la masse salariale. Juris association (juillet 2013) donne l'exemple d'une association de formation employant 170 salariés et s'acquittant d'une taxe sur les salaires annuelle de 322230 euros: elle 
pourra économiser 14000 euros, alors qu'une entreprise ayant les mêmes caractéristiques dans le secteur lucratif bénéficiera, elle, d'un crédit d'impôt de plus de 220000 euros en 2014. "L'augmentation de l'abattement de la taxe sur les salairesn'a d'effet que jusqu'à seize équivalents temps plein payés au Smic. »

En savoir plus: www.recma.org/node/3777.

\section{L'emploi associatif en danger}

Estiméà 314 millions d'euros par les finances publiques, cet allégement forfaitaire de taxe sur les salaires est officiellement destiné à soutenir l'emploi associatif, qui se révèle en effet menacé par la politique générale d'austérité. Pour Didier Minot, du Collectif des associations citoyennes, le secteur associatif doit s'attendre à la disparition de 30000 à 40000 emplois en 2014 en raison de la baisse annoncée (de l'ordre de 1,5 milliard d'euros) des dotations aux collectivités locales. Recherches et Solidarités évoque pour sa part un "léger rebond» de $0,3 \%$ de l'emploi associatif en juillet-aoûtseptembre 2013 grâce aux emplois d'avenir. En savoir plus: www.recma.org/node/3756.

\section{Le mythe des avantages fiscaux associatifs}

Les effets de l'austérité sont aggravés par "une distorsion de concurrence » entre le privé lucratif et le privé non lucratif, nuance le rapport remis le 12 décembre 2013 par la mission parlementaire (Yves Blein, Laurent Grandguillaume, Jérôme Guedj et Régis Juanico) sur l'impact de la mise en œuvre du Cice sur la fiscalité du secteur privé non lucratif. "Ces inadaptations pèsent d'autant plus sur les organismes à but non lucratif que la fiscalité qui s'applique à eux, basée principalement sur la taxe sur les salaires, est peuflexible et pénalisante pour la création d'emplois. » Les députés démontent ainsi le mythe de l'avantage fiscal associatif en affirmant que les associations fiscalisées « ont un taux d'imposition moyen réel largement audessus de la moyenne des autres entreprises » (page 47). Salué par l'Union des employeurs de l'ESS (Udes) et la Conférence permanente des coordinations associatives (CPCA), le rapport en appelle donc à une remise à plat du régime fiscal du secteur.

En savoir plus: www.recma.org/node/3850.

\section{Quand la fiscalité associative renvoie au périmètre de $1^{\prime} \mathrm{ESS}$}

Incidemment, le rapport sur l'impact de la mise en œuvre du Cice sur la fiscalité du secteur non lucratif repose la question du périmètre del'ESS. La septième proposition (page 52) de la mission parlementaire préconise en effet d'ouvrir la possibilitéà «tous les organismes agréés au titre de l'ESS » de bénéficier du régime fiscal du don et du mécénat. La CPCA affiche en revanche «sa plus grande réserve ": "compte tenu du périmètre inclusif de l'ESS tel que prévu dans le projet de loi ESS, une telle mesure serait très dommageable pour les associations, puisqu'elle entraînerait un effet d'aubaine important pour les sociétés commerciales et conduirait inexorablement à une remise en cause de ce régime fiscal du mécénat, pourtant indispensable à la diversification des ressources associatives » (CPCA, communiqué du 18 décembre 2013). Comme quoi, il existe bien des "spécificités méritoires " (François Bloch-Lainé) aux groupements de personnes, par opposition aux «associations decapitaux » (Gide, Cours d'économie politique, 1919). JORDANE LEGLEYE 